Supporting Information

\title{
Crystallization of Lithium Carbonate from Aqueous Solution: New Insights into Crystal Agglomeration
}

Wenchao Yang ${ }^{a}$, Ling Zhou ${ }^{a, *}$, Jiayu Dai ${ }^{a}$, Lina Zhou ${ }^{a, b}$, Meijing Zhang ${ }^{a, b}$, Chuang Xie $^{a, b}$, Hongxun Hao ${ }^{a, b}$, Baohong Hou ${ }^{a, b}$,Ying Bao ${ }^{a, b}$, Qiuxiang Yin ${ }^{a, b, *}$

aSchool of Chemical Engineering and Technology, State Key Laboratory of Chemical Engineering, Tianjin University, Tianjin 300072, People's Republic of China.

${ }^{\mathrm{b}}$ The Co-Innovation Center of Chemistry and Chemical Engineering of Tianjin, Tianjin University, Tianjin 300072, People's Republic of China.

*Ling Zhou.: Email: zhouling@tju.edu.cn

*Qiuxiang Yin.: Email: qxyin@tju.edu.cn 


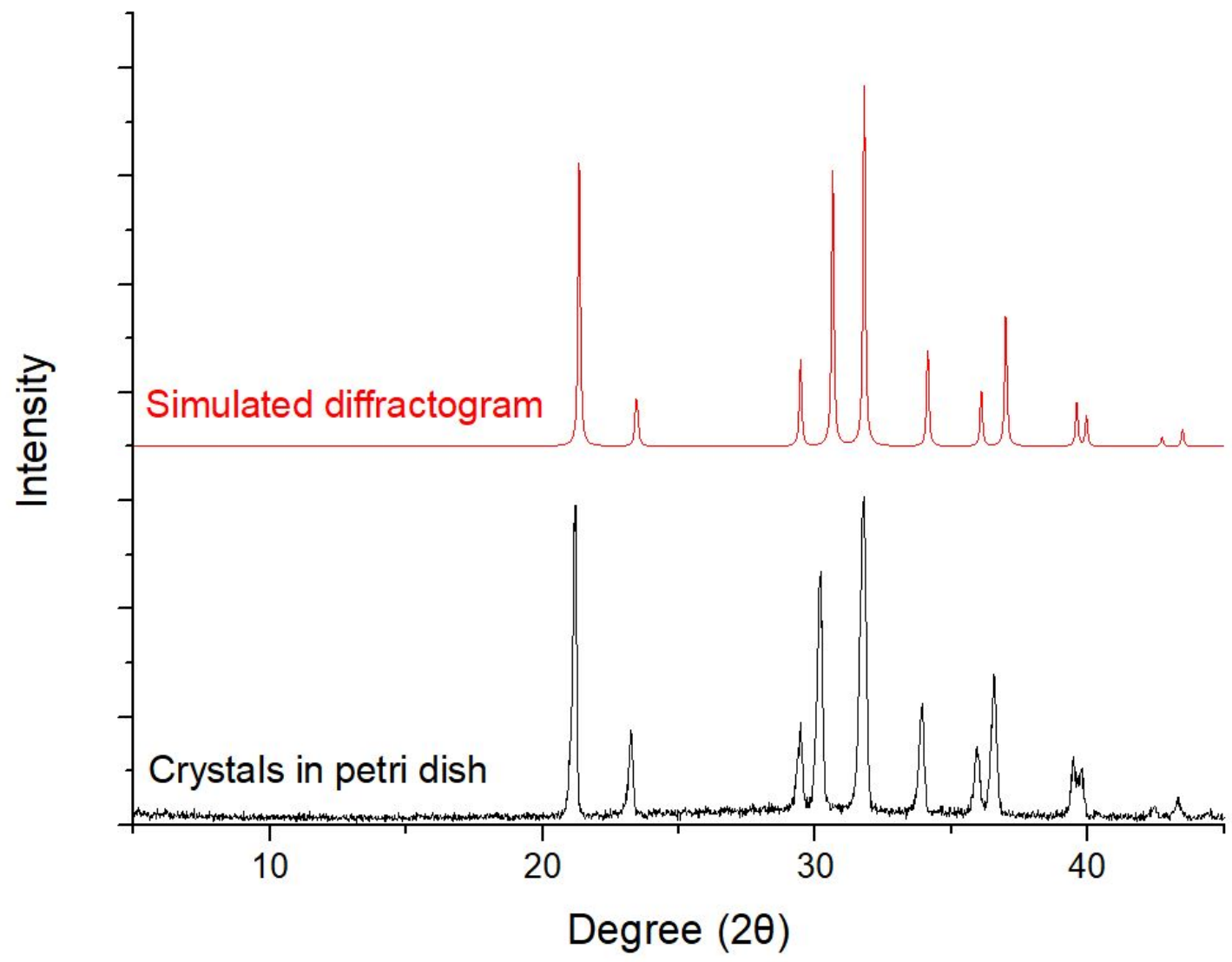

Figure S1. PXRD pattern of crystals in petri dish. 

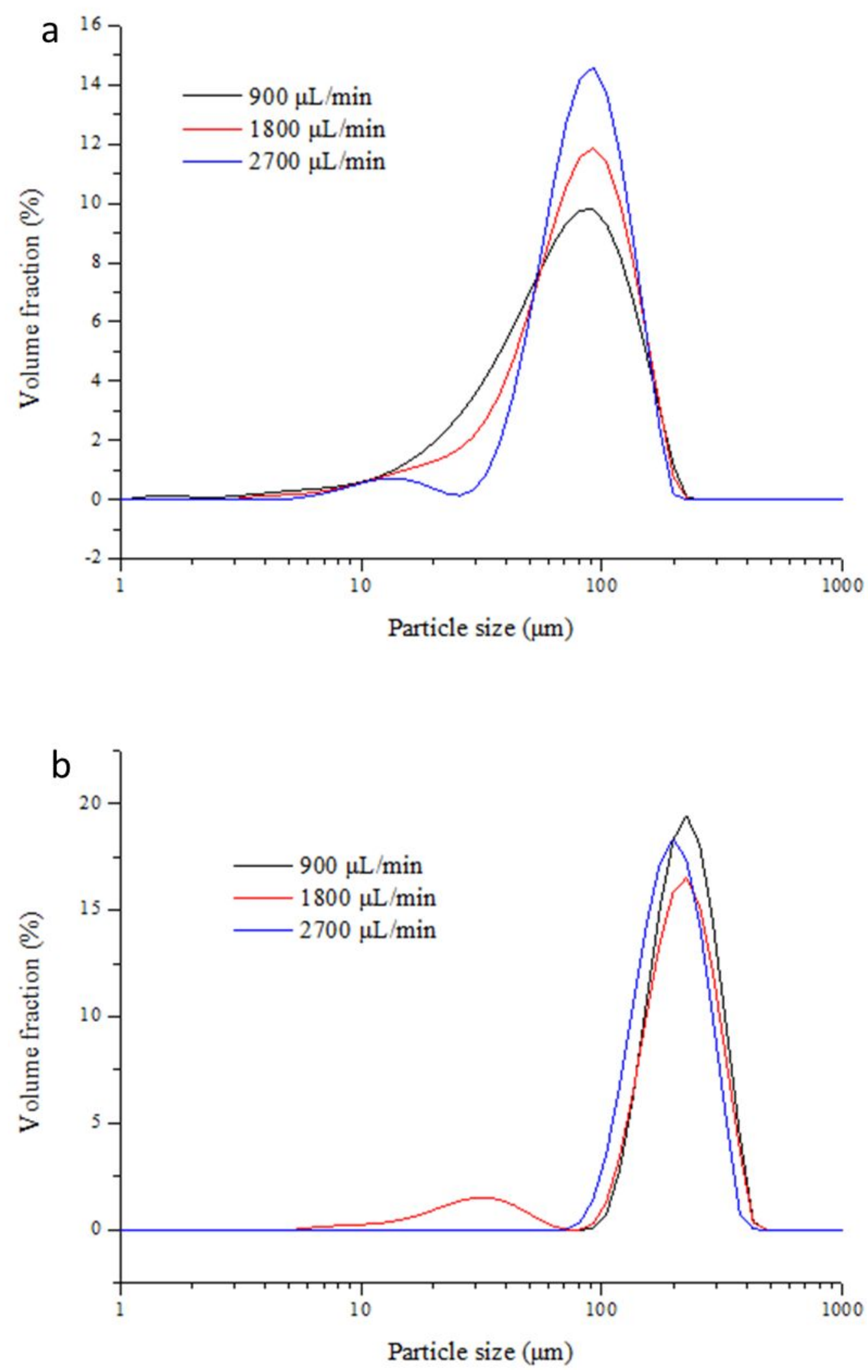

Figure S2. PSDs of lithium carbonate products under different adding rates: (a) needle-like crystals; (b) spherulites. 
Table S1. Amounts of $\mathrm{Na}$ and $\mathrm{K}$ in final products under different experimental conditions

\begin{tabular}{ccccc}
\hline \multirow{2}{*}{ Condition } & \multicolumn{2}{c}{$\mathrm{Na}(\%)$} & \multicolumn{2}{c}{$\mathrm{K}(\%)$} \\
& Mean & Std Dev & Mean & Std Dev \\
\hline 1 & 0.05 & 0.012 & 0.06 & 0.014 \\
2 & 0.13 & 0.014 & 0.12 & 0.013 \\
3 & 0.31 & 0.019 & 0.29 & 0.021 \\
5 & 0.07 & 0.011 & 0.06 & 0.012 \\
7 & 0.13 & 0.010 & 0.13 & 0.013 \\
\hline
\end{tabular}

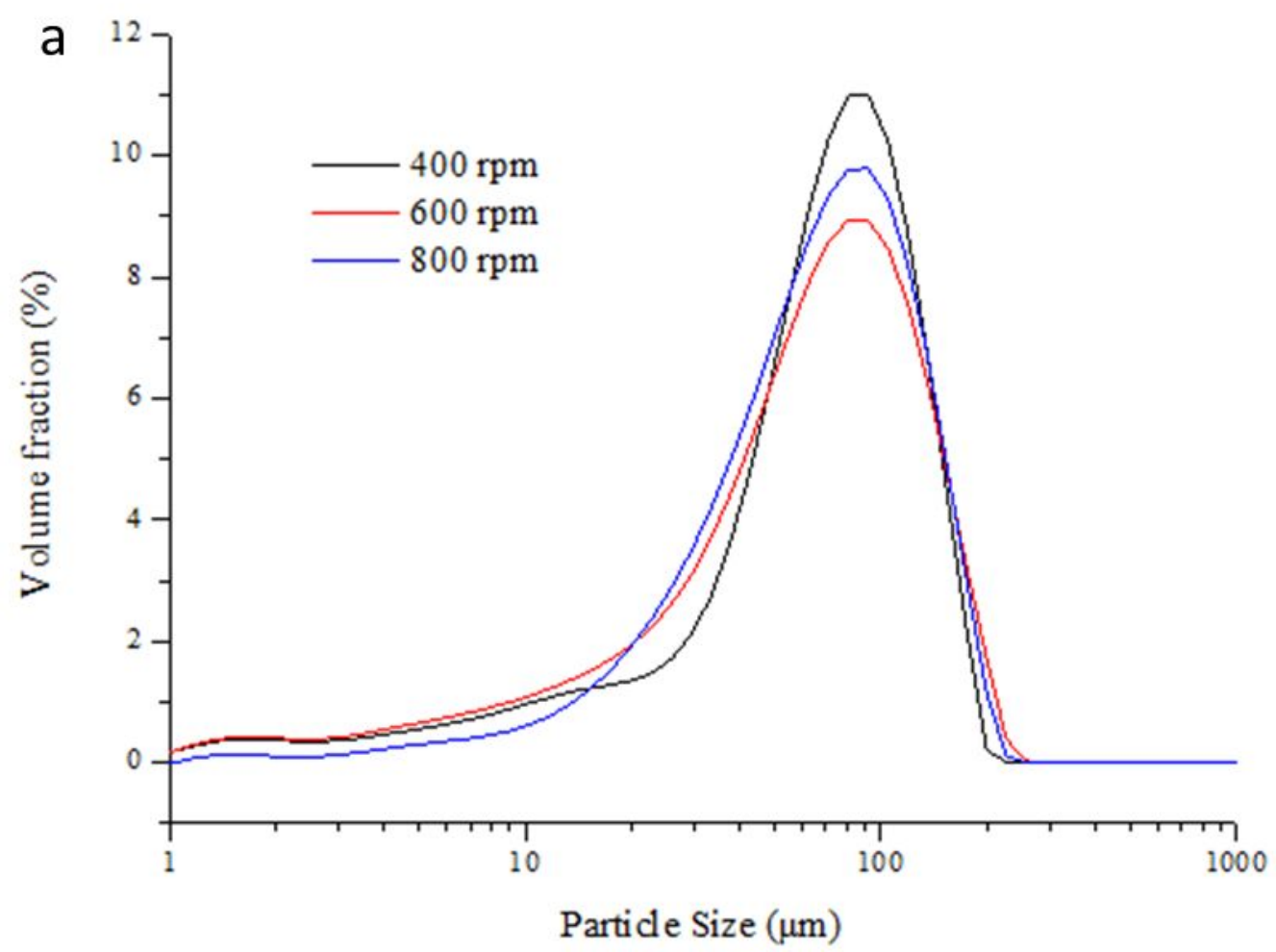




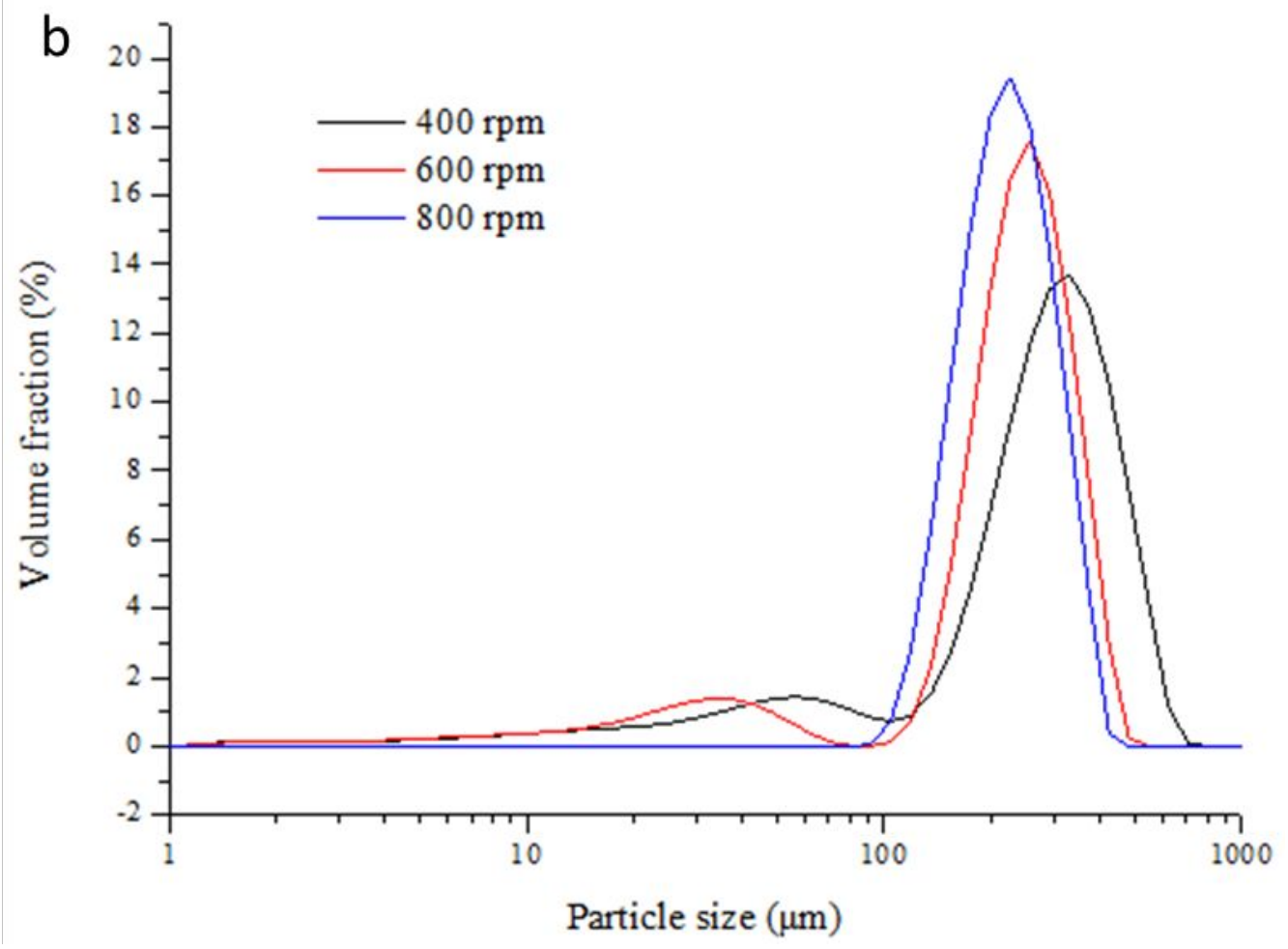

Figure S3. PSDs of lithium carbonate products under different agitation rates: (a) needle-like crystals; (b) spherulites.

Table S2. Data on $I_{C}$ calculation

\begin{tabular}{cccc}
\hline Condition & $\rho_{t}(\mathrm{~g} / \mathrm{ml})$ & $\rho_{b}(\mathrm{~g} / \mathrm{ml})$ & $I_{C}$ \\
\hline 8 & 0.81 & 0.69 & 0.15 \\
9 & 0.42 & 0.26 & 0.38 \\
\hline
\end{tabular}

